\title{
BMJ Open Engaging practices and communities in the development of interventions to promote HPV vaccine uptake: a protocol for implementing Boot Camp Translation in the private practice setting
}

\author{
Sarah E Brewer (D) ,1,2 Matthew J Simpson, ${ }^{1,2}$ John D Rice, ${ }^{1,3}$ Amanda Skenadore, ${ }^{1}$ \\ Sean T O'Leary ${ }^{1,4}$
}

To cite: Brewer SE, Simpson MJ, Rice JD, et al. Engaging practices and communities in the development of interventions to promote HPV vaccine uptake: a protocol for implementing Boot Camp Translation in the private practice setting. BMJ Open 2020;10:e041685. doi:10.1136/ bmjopen-2020-041685

- Prepublication history for this paper is available online. To view these files, please visit the journal online (http://dx.doi org/10.1136/bmjopen-2020041685).

Received 14 June 2020 Revised 17 November 2020 Accepted 25 November 2020

Check for updates

(C) Author(s) (or their employer(s)) 2020. Re-use permitted under CC BY-NC. No commercial re-use. See rights and permissions. Published by BMJ.

For numbered affiliations see end of article.

Correspondence to

Dr Sarah E Brewer;

sarah.brewer@cuanschutz.edu

\section{ABSTRACT}

Introduction The Healthy People 2020 report states a goal of $80 \%$ uptake of recommended vaccines among adolescents, including the human papillomavirus (HPV) vaccine. However, the rate of uptake of the HPV vaccine is estimated at $51 \%$ in 2018 , which leaves young people vulnerable to morbidity and mortality from preventable, HPV-related cancers. Reasons for this are multifactorial and include factors at the level of the provider, primary care practice, patient and family, and community. The development of interventions that are responsive to these multifactorial barriers in real-world settings is a priority. Boot Camp Translation (BCT) is a community-engaged approach to message development for translating evidence-based practices into clinics and communities. This project aims to (1) Engage practices and communities in the development of interventions to promote HPV vaccine uptake and (2) Evaluate the impact of the BCT-designed intervention on practice-level HPV vaccine initiation rates. We hypothesise that the BCT-designed intervention will increase the rate of HPV vaccine initiation in the practices.

Methods and analysis This study will implement HPVfocused BCT in three counties in Colorado with a below average county-level vaccination rate. Each BCT group will design a multipronged intervention targeted at patients, parents, providers and the general community to then be disseminated in the participating practices and communities over the subsequent 6 -month period. The long-term goal is to develop a replicable approach and low-cost method of increasing HPV vaccine uptake that is easily adaptable to different settings and sociodemographic contexts.

Ethics and dissemination This study is approved by the Colorado Multiple Institutional Review Board. Results will be disseminated through peer-reviewed manuscripts and conference presentations, as well as within Colorado practice-based research networks. Trial registration number NCT04279964
Strengths and limitations of this study

- This study applies an evidence-based community engagement strategy-Boot Camp Translation (BCT) - to the private practice setting with the goal to improve human papillomavirus (HPV) vaccine uptake.

- BCT produces interventions that are disseminationready, incorporate both practice and community context, and improve the acceptability of evidencebased interventions to individual communities.

- This study uses a robust matched case-control evaluation to assess the implementation and effectiveness of the BCT approach to increase HPV vaccination rates.

- Practices will not be randomised; however, clinics with similar demographics will be recruited to serve as matched controls.

\section{INTRODUCTION}

The human papillomavirus (HPV) vaccine is underused. Healthy People 2020 states a goal coverage level of $80 \%$ for all vaccines routinely recommended for adolescents. ${ }^{1}$ These adolescent vaccines include the tetanus-diphtheria-acellular pertussis (Tdap), meningococcal (MenACWY), HPV and influenza vaccines. Although coverage rates for all these vaccines have been rising steadily, only Tdap and MenACWY coverage levels have achieved the national goals, with coverage rates for 2018 at $89 \%$ and $86.6 \%$, respectively. ${ }^{2} \mathrm{HPV}$ vaccination levels are increasing at a substantially slower pace than the other adolescent vaccines. Series completion rate for 2018 is estimated at $51.1 \%$ for adolescents in the USA. ${ }^{2}$ These trends persist for Colorado, where HPV vaccine series completion 
rates are only $53.8 \%{ }^{3}$ HPV vaccines are preferentially recommended for adolescents aged 11-12 years to provide the vaccine well before exposure to HPV infection, which typically occurs shortly after sexual debut. ${ }^{4}$ Unfortunately, in USA, adolescents aged 11-12 years have the lowest levels of HPV vaccine utilisation compared with all other adolescent age categories. Thus, there is an urgent need to find mechanisms to increase HPV vaccination uptake among adolescents, particularly adolescents aged 11-12 years.

The public health benefits of HPV vaccination cannot be realised until higher coverage levels among adolescents are achieved. Modelling studies indicate that high levels $(80 \%-100 \%)$ of adolescent HPV vaccine utilisation can result in near eradication of genital warts, a $63 \%-86 \%$ reduction of anal cancers, a $76 \%-83 \%$ reduction in cervical cancer and a 56\%-74\% decrease in abnormal Papanicolaou smears among women, and an $86 \%$ reduction in HPV-associated genital cancers in men. ${ }^{5}$ With more than $80 \%$ of sexually active adults infected with HPV by age 50 years, HPV vaccination is a cost-effective public health intervention, and widespread use of the vaccine could save billions of healthcare utilisation dollars related to the management of HPV-related cancers. ${ }^{6}$ However, when lower levels of HPV vaccine utilisation are considered, such as that found among adolescents in the USA, the cost-effectiveness of HPV vaccination is significantly less favourable. ${ }^{7}$ Barriers to HPV vaccination are often contextual and involve factors at the level of the provider, patient/parent, practice and policy. Engagement of stakeholders at each of those levels is likely to improve uptake.

\section{Developing interventions to promote uptake}

Previous research has demonstrated that multifaceted interventions that target both the provider and the patient are more successful than single-pronged interventions for improving vaccine uptake. ${ }^{89}$ Furthermore, interventions that include active engagement by stakeholders tend to have more success than interventions with passive engagement. ${ }^{6}$ Thus, community engagement strategies have promise for the development and implementation of interventions to increase HPV vaccine uptake. By engaging multiple stakeholders, these approaches to intervention development are more comprehensive as they account for numerous factors at the level of the patient, practice and the broader community. There have been relatively few studies evaluating the impact of community engagement strategies on HPV vaccination. Several studies have implemented social marketing approaches to promoting HPV vaccination, ${ }^{10}{ }^{11}$ but while community-focused, these interventions are often not community-engaged. Other studies have implemented focus groups, but focus groups often lack the intensity and depth of engagement required to improve the translation of evidence-based interventions. ${ }^{12-14}$

Boot Camp Translation (BCT) is a communityengagement approach that was developed in the High Plains Research Network, a geographically defined practice-based research network (PBRN) in eastern Colorado, to improve the translation of medical evidence into communities. ${ }^{15}$ The genesis of BCT was a Centers for Disease Control and Prevention grant to improve colorectal cancer (CRC) screening in rural and frontier counties in eastern Colorado. ${ }^{16}$ First, community members gained expertise in CRC screening through a continuing education presentation, with substantial time for community members to interact with the medical expert and ask questions. Community members changed the technical language to make the topic more accessible to their rural communities. They changed words such as 'colorectal' to 'colon cancer' to make it easier to talk about in public. Given the complexity of the concept of 'screening', they eliminated this language and changed the title of the project to 'Testing to Prevent Colon Cancer'. By disseminating these messages through local channels and primary care practices, the programme reached $70 \%$ of the target population and resulted in a $10 \%$ increase in testing. The same approach has been replicated in another region of Colorado with an estimated $5 \%$ increase in testing. ${ }^{17}$

Since inception, BCT has been used numerous times to address a wide variety of health topics, including hypertension, diabetes, chronic pain, mental health, asthma and cardiovascular disease prevention. ${ }^{15} 1819$ The BCT focused on asthma resulted in (1) A set of messages to increase awareness of asthma and its symptoms, and (2) A provider toolkit for use in primary care practices. The provider toolkits reached 57 out of the 58 primary care practices in eastern Colorado and resulted in $79 \%$ of the practices initiating or increasing their use of spirometry in diagnosis and management, $40 \%$ of the practices increased the use of inhaled corticosteroids for symptom control, and $53 \%$ of the practices increased their implementation of asthma action plans. ${ }^{18}$ Additionally, as part of the Agency for Healthcare Research and Quality EvidenceNOW initiative to improve cardiovascular care, four communities in Colorado and New Mexico used BCTs to create a distinct set of products that were strongly influenced by the community context. ${ }^{20}$ Early results (study in progress) indicate that participating primary care practices had a preference for materials developed by their local BCT. ${ }^{21}$ Most recently, a pilot project in Colorado demonstrated the feasibility of using the BCT for addressing low uptake of HPV vaccination in two Colorado communities; however, this project was not designed to evaluate its effectiveness in increasing HPV vaccination rates. ${ }^{22}$

These BCTs have been in diverse communities in Colorado, usually with the State Networks of Colorado Ambulatory Practices and Partners (SNOCAP) consortium of PBRNs. ${ }^{19} 23$ BCT products have disseminated within primary care practices participating in projects as well as the broader community. BCT is effective because it accomplishes several key tasks that influence local uptake and implementation: (1) Formation of critical partnerships between academic researchers, local 
Table 1 Boot Camp Translation for HPV vaccination in private practice settings, trial specifications

\begin{tabular}{|c|c|}
\hline Data category & Information \\
\hline $\begin{array}{l}\text { Registry and trial } \\
\text { number }\end{array}$ & ClinicalTrials.gov: NCT04279964 \\
\hline Date of registration & 20 Februaryt 2020 \\
\hline $\begin{array}{l}\text { Secondary identifying } \\
\text { numbers }\end{array}$ & $18-0338$ \\
\hline Financial support & $\begin{array}{l}\text { National Cancer Institute, National } \\
\text { Institutes of Health }\end{array}$ \\
\hline Contact for queries & sean.oleary@cuanschutz.edu \\
\hline Title & $\begin{array}{l}\text { Engaging practices and communities } \\
\text { in the development of interventions to } \\
\text { promote HPV vaccine uptake }\end{array}$ \\
\hline Countries of recruitment & USA \\
\hline Health condition studied & HPV vaccination \\
\hline Intervention(s) & $\begin{array}{l}\text { Patient and provider engagement in } \\
\text { message development through Boot } \\
\text { Camp Translation }\end{array}$ \\
\hline $\begin{array}{l}\text { Key inclusion and } \\
\text { exclusion criteria }\end{array}$ & $\begin{array}{l}\text { Inclusion: patient aged } 11-17 \text { years } \\
\text { receiving care at participating practice } \\
\text { Exclusion: none }\end{array}$ \\
\hline Study type & Matched case-control \\
\hline Date of first enrolment & Anticipated 1 Octobert 2020 \\
\hline Target sample size & 2400 \\
\hline Trial status & Pre-intervention \\
\hline Primary outcomes & $\begin{array}{l}\text { Initiation of the HPV vaccine series } \\
\text { among adolescents aged } 11-12 \text { years }\end{array}$ \\
\hline $\begin{array}{l}\text { Key secondary } \\
\text { outcomes }\end{array}$ & $\begin{array}{l}\text { Completion of the HPV vaccine series } \\
\text { among patients aged } 11-12 \text { years } \\
\text { Completion of the HPV vaccine series } \\
\text { among patients aged } 13-17 \text { years }\end{array}$ \\
\hline
\end{tabular}

HPV, human papillomavirus.

community leaders and primary care practice champions, ${ }^{24}{ }^{25}$ (2) Increasing local relevance of an evidencebased intervention by using language and constructs that resonate locally, and (3) Disseminating the new messages through effective community channels. ${ }^{15}$

This project aims to implement a proven engagement methodology to translate the current guidelines and evidence for HPV vaccination into contextually relevant interventions that will accelerate the adoption of the HPV vaccine. This approach can easily be scaled throughout the state of Colorado with our network of primary care PBRNs, ${ }^{23} 2627$ partnerships with public health departments and numerous community partners. Furthermore, this study will test a novel approach to the development of relevant interventions to promote HPV vaccine uptake that can subsequently be replicated across other PBRNs in the USA.

\section{METHODS}

Trial specifications are described in table 1.

\section{Study design}

The first main goal of the project will be to implement the BCT method in three geographically distinct Colorado counties to translate the current evidence for HPV vaccination into locally relevant messages designed to increase HPV vaccine uptake. We will then disseminate the developed messages in the participating practices.

The second main goal of the project will be to evaluate the impact of the BCT-designed intervention on practicelevel HPV vaccine initiation rates. We hypothesise that the BCT-designed intervention will increase the rate of HPV vaccine initiation in those practices where it has been implemented, relative to matched control practices. We seek to determine the difference in vaccine initiation rates among adolescents aged 9-17 years between control and intervention practices over the practice implementation period (6 months).

\section{Patient and public involvement}

This study is based on the assumption that patient and community involvement in research, from design through dissemination, improves the research process and health outcomes. The BCT method engages patients, healthcare providers and other stakeholders in designing, implementing, evaluating and disseminating messaging interventions for HPV vaccination. Patents and practice staff will be involved in all decision-making about each of these stages of the research.

\section{Participants}

\section{Clinics}

We will identify three geographically distinct (nonneighbouring) counties with lower HPV vaccination rates than the state average for our recruitment. From there, we will recruit six primary care clinics (two from each county) from a statewide PBRN consortium. The SNOCAP consortium consists of over 100 family medicine practices and over 50 paediatric primary care practices distributed geographically across the state. Two recruited practices will be in rural Colorado. Family medicine and paediatric clinics will be represented. Once the clinics confirm participation, Business Associates Agreements and Data Use Agreements will be drafted and signed as appropriate. Active bidirectional use of the Colorado Immunization Information System (CIIS) will be required for practice inclusion.

\section{BCT participants}

We will target approximately 10 Community Advisory Group members per BCT. BCT Community Advisory Group members have local community expertise. Identification and engagement of BCT Community Advisory Group members will use the same processes that have been successful in previous BCT projects. ${ }^{12}{ }^{1315-19}$ As we will be recruiting two primary care clinics for participation, we will solicit participation from two clinic team members (one clinician, one medical assistant or nurse) from each clinic. The remaining six participants will be 


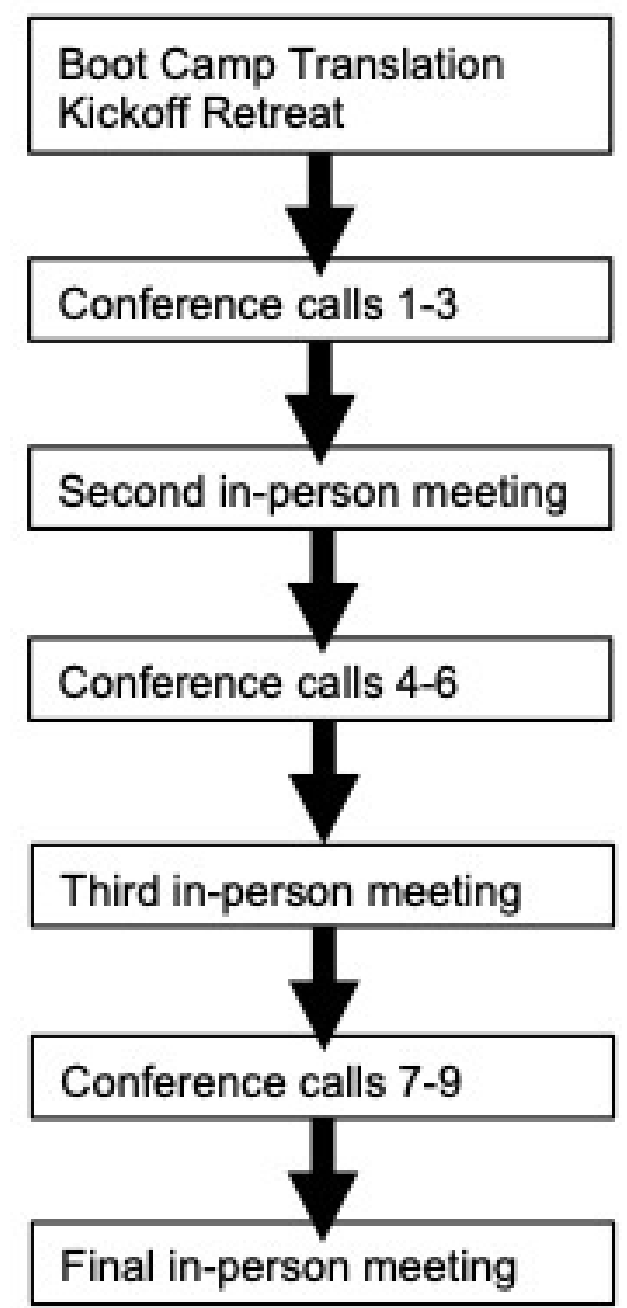

Figure 1 The Boot Camp Translation process.

recruited from key stakeholder groups, to include parents of adolescents, adolescents, local public health departments, and cancer advocates or survivors. Clinics will be encouraged to recruit patients and parents for participation. BCT Community Advisory Group members will be asked to commit to a 6-month process.

\section{Adolescent patients}

All child patients who have received health supervision at participating practices during the 12 months prior to intervention and the 12 months following intervention implementation, who are aged 9-17 years and have not already initiated the HPV vaccine series, and whose parents have not requested removal from the CIIS will be eligible for the assessment of practice immunisation rates in the pre and post periods.

\section{Intervention}

The BCT process is summarised in figure 1. BCT begins with a daylong retreat where a member of the project team gives a presentation on adolescent vaccination, and BCT Community Advisory Group members have ample time to have all their questions about the topic answered. The BCT facilitator then works with the group to address two questions: (1) What does our message to the community about HPV vaccination need to say? and (2) How do we disseminate that message to our practices and community? In the subsequent $7-10$ conference call meetings and three inperson meetings, the facilitation team guides the BCT Community Advisory Group members towards the generation of 'products' (pamphlets, posters, ads, articles, talks, clinician aids, etc) that resonate in the community, and corresponding implementation plans. These products convey the key messages about the evidence for HPV vaccination and can include new messages, new messaging strategies, or existing messages translated to a new setting or population within the target community. Throughout these meetings, further brainstorming will inevitably occur and be incorporated.

Given the context of the COVID-19 pandemic and the corresponding restrictions on gatherings, the BCT process will be adapted to be conducted virtually, with all participants attending the retreat, conference calls and other meetings via online videoconferencing. In addition, facilitators will lead discussions about how messaging and dissemination strategies will accommodate the COVID-19 restrictions in the practices and community setting. Finally, facilitators will work with each BCT group to incorporate appropriate contingencies for changes in the local COVID-19 context to optimise their dissemination plans. For example, groups may discuss what to do in the event of a surge in cases and increased local restrictions; or conversely, what additional dissemination strategies might be employed if case rates fall and restrictions are lifted. These decisions will guide the implementation by the research team after the completion of the BCT process.

\section{Sample size and power calculations}

Intent-to-treat analyses will be conducted to test the primary study hypothesis that patients in intervention practices who have not previously received at least one dose of the HPV vaccine will be more likely to have done so by the end of the 6-month intervention period (12 months postbaseline, with the 6-month Boot Camp process as a washout period) than those in control practices. We estimate that each participating practice will have a minimum of 200 adolescents during the 6-month intervention period that have not previously received the HPV vaccine. We will use practice size and number of providers as a proxy for number of adolescent patients ${ }^{28}$ and estimate a $30 \%-50 \%$ vaccination rate for those adolescents. Based on these estimates, practices with at least two providers will be eligible for participation.

We based our power calculations on vaccine utilisation rates in control practices of $50 \%$, since this is the most conservative proportion for calculating sample size (ie, requires the largest sample to detect differences between experimental arms). Our final sample size for analysis would be 2400 patients in 12 practices, 6 intervention and 6 matched control practices. At this sample size, we will have $80 \%$ power to detect an effect size of $9.8 \%$ for an 
intraclass correlation coefficient of 0.01 , and $90 \%$ power for an effect size of $11.3 \%$. This corresponds, respectively, to vaccine utilisation rates in intervention practices of $59.8 \%$ and $61.3 \%$. As this is a preliminary study with a 2-year timeline, we have chosen to compare intervention practices to matched controls rather than implement a randomised design, as randomisation would double the number of counties we would need to participate.

\section{Measures}

Evaluating implementation of BCT-designed interventions

In the participating practices, we will use the RE-AIM (reach, effectiveness, adoption, implementation, and maintenance) framework ${ }^{29-31}$ to assess the reach, adoption, implementation and maintenance of the intervention over the 6-month intervention period. Additionally, we will seek to assess attribution of changes in vaccine initiation rates. Evaluation will be tailored to the specific intervention, delivery modality and implementation strategy developed in the BCT. We anticipate that we will conduct five to seven parent and practice staff interviews per practice site and conduct a survey of a random sample of 30-40 parents of HPV-vaccine eligible patients per practice to assess each of the RE-AIM domains. Surveys will assess exposure to the intervention, impact of the intervention message and acceptability of the intervention. We will also document implementation and maintenance activities undertaken by the research team and practice staff throughout the practice implementation phase. These data will serve to help explain findings related to vaccine initiation (Aim 2) and inform the translation of this intervention approach to a larger trial.

\section{Outcomes}

Outcomes generated from the BCT process will include, for each practice, (1) A set of core messages, (2) Communication materials, and (3) A community implementation and dissemination strategy. The research team, along with the BCT Community Advisory Group members, will present these materials and plans to the relevant practices who will work with the project research team in Aim 2 to implement the messages and materials of the plan.

The primary outcome for the matched case-control phase of the study will be binary, with a success counted if a vaccine-eligible adolescent patient aged 9-17 years has received at least one HPV vaccine over the follow-up period. Vaccine-eligible participants will be defined as patients in the participating practices aged 9-26 years who have not previously received an HPV vaccine as recorded in the CIIS. Secondary analyses will investigate the difference in initiation rates between intervention and control practices in boys compared with girls as well as different age strata (9-10, 11-12 and 13-17 years). Additionally, for the participating practices that see adult patients, we will investigate the difference in vaccine initiation rates between intervention and control practices among eligible adults aged 18-26 years.

\section{Data sources}

Participating practices will complete a practice descriptive, which will include practice members, size, ownership, patient population, number of clinicians and specialty of the clinicians. The following types of data will be obtained from the CIIS: patient-level immunisation data, including the number, date and type of specific vaccines received; patient-level demographic data, including age, gender and race/ethnicity. Control practices will be chosen to match intervention practices on size (total full time equivalent (FTE)), per cent of patients using Vaccines for Children, setting (urban/suburban/rural) and baseline HPV initiation rates. Control practices must also be active CIIS users; we will obtain baseline rates of initiation for practices in several counties that are similar to our chosen study counties in terms of demographics and size, and then choose from the practices within those counties that have similar baseline rates to our intervention practices. The design requires only that we obtain CIIS data for the control practices, with minimal associated increases in effort. CIIS data are highly accurate, with frequent quality checks.

\section{Data collection methods}

The subjects of this study include the clinic providers and practice staff members of the participating clinics and their patients and the parents of patients that are eligible for participation in the study. Additionally, each BCT group will include clinic providers and staff as well as local community members, all of whom will be participating in the study. For all data obtained about providers, clinic staff members, patients, parents of patients and community members, we will use confidentiality standards to protect them and their data.

\section{Assessment of immunisation status}

The primary analysis will be a comparison of HPV vaccine initiation between the control and intervention practices, defined as the proportion of eligible patients aged 9-17years who are unvaccinated at the start of the study but who initiate vaccination before its end. Secondary analyses will examine differences in HPV vaccine initiation based on patient characteristics: age (including patients aged 18-26years), sex and race/ethnicity. Additionally, we will explore through multivariable analyses whether practice type (public vs private), medical specialty, payer mix, patient sociodemographics and number of providers impacts the effectiveness of the intervention. In order to address the possibility of differential effects of the intervention by site, we will conduct secondary analyses including a random slope for treatment in the model.

\section{Statistical analysis}

In this pre-experimental pilot study, descriptive statistics will be computed for patient and practice characteristics in both control and intervention practices. Patient-level and practice-level covariates will be screened in bivariable analyses and included in multivariable analysis if they are 
related to the outcome at $\mathrm{p}<0.2$ or differ between treatment arms. Covariates and potential moderators at the patient level will include: age, gender and race/ethnicity.

We will employ intent-to-treat analyses using generalised linear mixed models (GLMMs) to account for the clustering of patients within practices as well as the matching of controls to intervention practices, assuming ignorable missingness (missing completely at random or missing at random) ${ }^{32}{ }^{33}$ Hypothesis tests will be two-sided with a significance level at $\alpha=0.05 ; p$ values will also be reported. All statistical analyses will be performed using SAS V.9.4 (SAS Institute, Cary, North Carolina, USA).

The primary independent variable for all analyses will be intervention status of the practice. For overall analyses with the 12 sites we plan to include a random intercept for each practice $j=1,2$ within matched pairs and for each matched pair $k=1, \ldots, 6$. We will therefore use a hierarchical logistic regression model:

$$
\log \frac{\pi_{i j k}}{1-\pi_{i j k}}=\beta_{0}+\beta_{1}(j-1)+v_{k}+u_{j k}
$$

where $\pi_{i j k}$ is the probability of initiating vaccination for subject $i$ within practice $j$ in pair $k, \beta_{1}$ is the treatment effect $(\log$ OR of initiation for intervention practices relative to control practices), $j-1$ is intervention indicator (assuming practice 1 in each matched pair is control and 2 is intervention), and $v_{k}$ and $u_{i k}$ are the pair and practice random effects, respectively, (assumed to be jointly normally distributed). Additional patient-level and practice-level covariates will be included in the model as described above. To examine the effects of the intervention on time to vaccination initiation, Cox models will be fit to the data, where the outcome is time from beginning of the study to vaccine initiation or the end of the study (censoring), whichever is first. A test of the HR associated with the treatment indicator variable will allow for determination of whether the intervention significantly affected time to initiation. ${ }^{34}$

\section{ETHICS AND DISSEMINATION Informed consent}

There will be no direct contact with patients in this study. Instead, participation will consist only of retrospective review of their CIIS vaccination records. These data will be aggregated at the practice level and will contain deidentified patient data. A HIPAA (Health Insurance Portability and Accountability Act of 1996) waiver and a waiver of documentation of written consent for this aspect of the study have been approved.

Other data will be collected for the purpose of the research only. Surveys conducted with providers, staff members, parents of patients and community members will include a postcard consent in which information is provided and the participant consents by completing the survey. They are also free to decline and not complete the survey. Another form of data collected for this study are interviews with providers, practice staff members, parents of patients, and community members. These activities will only commence once the participant has completed a verbal consent agreement.

\section{Consent and confidentiality procedures}

The subjects of this study include the practice providers and practice staff members of the participating practices and their patients and the parents of patients that are eligible for participation in the study. Additionally, each BCT group will include practice providers and staff as well as local community members, all of whom will be participating in the study. For all data obtained about providers, practice staff members, patients, parents of patients and community members, we will use confidentiality standards to protect them and their data.

To maintain privacy, as providers and patients are identified in practices, we will generate a study identification (ID) code for each person. Data generated by the researchers or research assistant for analysis will be by study ID code only and not be identified by name. We will create a match list of names/contact information with study assigned ID codes to be maintained. This list will be kept in a password protected computerised file and not shared with others beyond the study investigators and research assistant. Data will not be reported on an individual's results, but instead summarised to provide group data.

\section{Monitoring}

The principal investigator will have overall responsibility for participant safety monitoring. The risks for this intervention development and implementation of this behavioural intervention are minimal. Oversight for data safety and monitoring will be conducted by a faculty member at the University of Colorado, Denver, who is not involved in the project. In this capacity, the individual will provide independent observation and verification of protocol compliance, recruitment and study progress, and data completeness. The data safety monitor (DSM) will monitor the study for adverse events, and the study team's response to these events, should any occur. Though adverse events are not anticipated, they will be reported to the institutional review board and the DSM at the time of the event should any occur.

\section{Assessment of immunisation status}

The nature of the data collection for this study includes data obtained from the CIIS and electronic medical record (EMR) to select a random sample of patients to participate in surveys to evaluate the intervention. The EMR data will consist of data collected in the course of patient care and includes the patient's demographic information. For these demographic data, collected in the course of standard care delivery, patients will not be approached for consent as their information will only be provided as contact information to invite patients to participate in the survey. Only the healthcare personnel in the clinic or in a business associate role (ie, chart abstractor) will know the identity of the patients. These data will be coded for 
confidentiality using a study ID code and be securely transferred using appropriate protocols for secure data transfer. We will apply for a waiver of consent and HIPAA waiver of authorisation for this portion of our data collection that are consistent with the nature of a limited data set.

\section{Access to data}

Access to data will be limited to the research team. Access to a deidentified, aggregated version of the data set and analysis code will be available on request with approval by the research team.

\section{Dissemination plans}

With this exploratory pre-experimental study, we seek to use BCT to develop interventions that promote the uptake of HPV vaccination and determine preliminary effect size estimates. The results of this study can then be disseminated to practices nationally and through the PBRNs in Colorado by recruiting more practices to serve as study sites to implement BCT-generated materials. The materials generated in this study will be combined with the previously designed community-based materials and made available to other primary care practice and communities. Furthermore, our advisory group will serve to inform the dissemination in Colorado with a specific focus on incorporating the BCT materials into other initiatives within Colorado to promote HPV vaccine uptake. We also plan to share the findings of this study in scientific presentations and publications, as well as through workshops at our annual PBRN convocation and monthly online newsletter.

\section{DISCUSSION}

To address suboptimal HPV vaccination rates, this study applies an evidence-based community engagement strategy $-\mathrm{BCT}$ - in the private practice setting to engage patient stakeholders alongside private practice staff to develop messaging and interventions promoting the HPV vaccine. The BCT approach produces interventions that are dissemination-ready, incorporate both practice and community context, and improve the acceptability of evidence-based interventions to individual communities. This study will use a robust matched case-control evaluation to assess the implementation and effectiveness of the BCT approach to increase HPV vaccination rates among adolescents aged 9-17 years. While practices will not be randomised, clinics with similar demographics will be recruited to serve as matched controls. Ultimately, we anticipate this study will demonstrate the feasibility and effectiveness of using community-engaged strategies at the private clinic level to improve HPV vaccination rates and prevent HPV-related cancers.

\section{Author affiliations}

${ }^{1}$ Adult and Child Consortium for Health Outcomes Research and Delivery Sciences (ACCORDS), University of Colorado Anschutz Medical Campus, Aurora, CO, USA
${ }^{2}$ Family Medicine, University of Colorado Anschutz Medical Campus, Aurora, CO, USA

${ }^{3}$ Biostatistics and Informatics, Colorado School of Public Health, University of Colorado Anschutz Medical Campus, Aurora, CO, USA

${ }^{4}$ Pediatrics, University of Colorado Anschutz Medical Campus, Aurora, C0, USA

Correction notice The article has been corrected since it was published. The author name, Amanda Skenadore has been updated.

Contributors SEB, MJS, JDR and STO conceived of the study and intervention and wrote the study protocol. SEB wrote the first draft of the manuscript. MJS, JDR, AS and STO provided input into the study design, intervention development and study protocol, and edited and/or reviewed the manuscript.

Funding This work was supported by the National Cancer Institute at the US National Institutes of Health, grant number R21CA230878-01A1.

\section{Competing interests None declared.}

Patient and public involvement Patients and/or the public were involved in the design, or conduct, or reporting, or dissemination plans of this research. Refer to the Methods section for further details.

Patient consent for publication Not required.

Provenance and peer review Not commissioned; externally peer reviewed.

Open access This is an open access article distributed in accordance with the Creative Commons Attribution Non Commercial (CC BY-NC 4.0) license, which permits others to distribute, remix, adapt, build upon this work non-commercially, and license their derivative works on different terms, provided the original work is properly cited, appropriate credit is given, any changes made indicated, and the use is non-commercial. See: http://creativecommons.org/licenses/by-nc/4.0/.

ORCID iD

Sarah E Brewer http://orcid.org/0000-0003-0063-6626

\section{REFERENCES}

1 Office of Disease Prevention and Health Promotion. Immunization and infectious diseases, 2017. Available: https://www.healthypeople. gov/2020/topics-objectives/topic/immunization-and-infectiousdiseases/objectives [Accessed 6 Oct 2017].

2 Walker TY, Elam-Evans LD, Yankey D, et al. National, Regional, State, and Selected Local Area Vaccination Coverage Among Adolescents Aged 13-17 Years - United States, 2018. MMWR Morb Mortal Wkly Rep 2019;68:718-23.

3 Colorado Department of Public Health and Environment. CIIS County level rates 2019, 2019. Available: https://www.colorado.gov/pacific/ cdphe/ciiscountylevel [Accessed 3 Oct 2019].

4 HPV vaccine recommendations. Pediatrics: Committee on Infectious Diseases 2012:602-5.

5 Van de Velde N, Boily M-C, Drolet M, et al. Population-Leve impact of the bivalent, quadrivalent, and nonavalent human papillomavirus vaccines: a model-based analysis. J Natl Cancer Inst 2012;104:1712-23.

6 Seto K, Marra F, Raymakers A, et al. The cost effectiveness of human papillomavirus vaccines: a systematic review. Drugs 2012;72:715-43.

7 Canfell K, Chesson H, Kulasingam SL, et al. Modeling preventative strategies against human papillomavirus-related disease in developed countries. Vaccine 2012;30:F157-67.

8 Fiks AG, Grundmeier RW, Mayne S, et al. Effectiveness of decision support for families, clinicians, or both on HPV vaccine receipt. Pediatrics 2013;131:. :1114-24.

9 Cassidy B, Braxter B, Charron-Prochownik D, et al. A quality improvement initiative to increase HPV vaccine rates using an educational and reminder strategy with parents of preteen girls. $J$ Pediatr Health Care 2014;28:155-64.

10 Cates JR, Coyne-Beasley T. Social marketing to promote HPV vaccination in pre-teenage children: talk about a sexually transmitted infection. Hum Vaccin Immunother 2015;11:347-9.

11 Cates JR, Diehl SJ, Crandell JL, et al. Intervention effects from a social marketing campaign to promote HPV vaccination in preteen boys. Vaccine 2014;32:4171-8.

12 Brawner BM, Baker JL, Voytek CD, et al. The development of a culturally relevant, theoretically driven HPV prevention intervention for urban adolescent females and their parents/guardians. Health Promot Pract 2013;14:624-36.

13 Katz ML, Paskett ED. The process of engaging members from two underserved populations in the development of interventions 
to promote the uptake of the HPV vaccine. Health Promot Pract 2015;16:443-53.

14 Head KJ, Cohen EL. Young women's perspectives on cervical cancer prevention in Appalachian Kentucky. Qual Health Res 2012;22:476-87.

15 Westfall JM, Zittleman L, Felzien M, et al. Reinventing the wheel of medical evidence: how the boot cAMP translation process is making gains. Health Aff 2016;35:613-8.

16 Zittleman L, Emsermann C, Dickinson M, et al. Increasing colon cancer testing in rural Colorado: evaluation of the exposure to a community-based awareness campaign. BMC Public Health 2009;9:288

17 Westfall JM, Zittleman L, Sutter C, et al. Testing to prevent colon cancer: results from a rural community intervention. Ann Fam Med 2013;11:500-7.

18 Bender BG, Dickinson P, Rankin A, et al. The Colorado asthma toolkit program: a practice coaching intervention from the high plains research network. J Am Board Fam Med 2011;24:240-8.

19 Norman N, Bennett C, Cowart S, et al. Boot cAMP translation: a method for building a community of solution. J Am Board Fam Med 2013;26:254-63.

20 English AF, Dickinson LM, Zittleman L, et al. A community engagement method to design patient engagement materials for cardiovascular health. Ann Fam Med 2018;16:S58-64.

21 North American Primary Care Research Group Annual Meeting. Practice preferences for regional boot cAMP translation produced materials: results from four boot CAMP translations for EvidenceNOW Southwest. Chicago, IL, 2018.

22 Brewer SE, Nederveld A, Simpson M. Engaging communities in preventing human papillomavirus-related cancers: two boot CAMP translations, Colorado, 2017-2018. Preventing Chronic Disease 2020:17.

23 Fisher M, Brewer SE, Westfall JM, et al. Strategies for developing and sustaining patient and community Advisory groups: lessons from the state networks of Colorado ambulatory practices and partners (SNOCAP) Consortium of practice-based research networks. J Am Board Fam Med 2019;32:663-73.
24 Brown CH, Kellam SG, Kaupert S, et al. Partnerships for the design, conduct, and analysis of effectiveness, and implementation research: experiences of the prevention science and methodology group. Adm Policy Ment Health 2012;39:301-16.

25 Kellam SG. Developing and maintaining partnerships as the foundation of implementation and implementation science: reflections over a half century. Adm Policy Ment Health 2012;39:317-20.

26 Fisher M, Brewer SE, Fernald DH, et al. Process for setting research priorities: a case study from the state networks of Colorado ambulatory practices and partners (SNOCAP) Consortium. J Am Board Fam Med 2019;32:655-62.

27 Brewer SE, Crump NM, O'Leary ST. Patient-Centered research priorities: a mixed-methods approach from the Colorado children's outcomes network (COCONet). J Am Board Fam Med 2019;32:674-84.

28 Bocian AB, Wasserman RC, Slora EJ, et al. Size and age-sex distribution of pediatric practice: a study from pediatric research in office settings. Arch Pediatr Adolesc Med 1999;153:9-14.

29 Glasgow RE, Vogt TM, Boles SM. Evaluating the public health impact of health promotion interventions: the RE-AIM framework. Am J Public Health 1999;89:1322-7.

30 Glasgow RE, Klesges LM, Dzewaltowski DA, et al. Evaluating the impact of health promotion programs: using the RE-AIM framework to form summary measures for decision making involving complex issues. Health Educ Res 2006;21:688-94.

31 Glasgow RE, Harden SM, Gaglio B, et al. RE-AIM planning and evaluation framework: adapting to new science and practice with a 20-year review. Front Public Health 2019;7:64.

32 Diggle PJ, Heagerty P, Liang K-Y, et al. Analysis of longitudinal data: Oxford university press 2002.

33 Reagan-Steiner S, Yankey D, Jeyarajah J, et al. National, Regional, State, and Selected Local Area Vaccination Coverage Among Adolescents Aged 13-17 Years-United States, 2014. MMWR Morb Mortal Wkly Rep 2015;64:784-92.

34 Kalbfleisch JD, Prentice RL. The statistical analysis of failure time data: John Wiley \& Sons 2011. 